\title{
An operator-based exact treatment of open quantum systems
}

\author{
S.Nicolosi* \\ stefania.nicolosi@virgilio.it \\ INFM, MIUR and Dipartimento di Scienze Fisiche ed Astronomiche, via Archirafi 36, 90123 \\ Palermo, Italy, Tel: +39091 6234248, Fax: +390916234281
}

\begin{abstract}
Quantum mechanics must be regarded as open systems. On one hand, this is due to the fact that, like in classical physics, any realistic system is subjected to a coupling to an uncontrollable environment which influences it in a non-negligible way. The theory of open quantum systems thus play a major role in many applications of quantum physics since perfect isolation of quantum system is not possible and since a complete microscopic description or control of the environment degrees of freedom is not feasible or only partially so [1]. Practical considerations therefore force one to seek for a simpler, effectively probabilistic description in terms of an open system. There is a close physical and mathematical connection between the evolution of an open system, the state changes induced by quantum measurements, and the classical notion of a stochastic process. The paper provides a bibliographic review of this interrelations, it shows the mathematical equivalence between markovian master equation and generalized piecewise deterministic processes [1] and it introduces the open system in an open observed environment model.
\end{abstract}

\section{Introduction}

The dynamics of open quantum systems plays a central role in a wide class of physical systems. Usually, the dynamics of an open system is described in terms of the reduced density matrix $\rho_{S}(t)$ which is defined by the trace over the environment degrees of freedom $[1,2,3]$. On the ground of the weak-coupling assumption and the Rotating Wave Approximation the dynamics may be formulated in terms of a quantum dynamical semigroups which yields a Markovian Master Equation [4]. However, the dynamical equation, thus obtained, is very often untractable. This fact has encouraged some physicists $[1,5,6,7]$ to look for alternative ways to describe open systems. Instead of representing the dynamics of an open system by a quantum Master Equation for its density matrix, it is formulated in terms of a stochastic process for the open system's wave function. The stochastic representation of quantum Markov processes already appeared in a fundamental paper by Davies [8] and was applied [9] to derive the photocounting formula. While the theory was originally formulated in terms of a stochastic process for the reduced density matrix, in the last decade it has been proposed $[1,5,6,7]$ as a stochastic

1 thank Prof.N.Messina and Dr.A.Napoli to introduce me on the subject and to give me the freedom to follow my personal scientific interest. 
evolution of the state vector in the reduced Hilbert space (for a review see [10]). At the same time Carmichael has developed the idea of an unravelling of the master equation in terms of an ensemble of quantum trajectories. His theory is applicable only to a particular class of quantum systems (the photoemissive sources) and it induces to think that this treatment is equivalent to the Master Equation approach. In the last few years F.Petruccione and H.P.Breuer [1] generalize and provide a mathematical formulation of Carmichael's idea of quantum trajectories [5] and of the Monte Carlo wave function method [6]. In this contest their main result is to demonstrate that the dynamics given by the most general Master Equation in Lindblad form can be represented as a piecewise deterministic process (PDP) [1] $\psi(t)$ in the Hilbert space of the open system. The physical basis to achieve their aim is provided by continuous measurement theory [1].

The link [11] between the first and second way of describing open quantum systems is, however, only one way in the sense I am going to explain. The cited paper contains a flow diagram that is a clear and straightforward picture to review Concept and methods in the theory of open quantum systems, as the same authors title their work. The diagram shows us that PDPs, describing a selective level of measurement, implies the non-selective one, appearing in the form of a Lindblad markovian master equation, while the opposite has not been demonstrated. My aim is to put an arrow in the opposite direction in order to demonstrate the equivalence between the two approaches to open systems dynamics, at least under Born-Markov approximation. To achieve my goal I start from microscopic models and, exploiting the same approximation leading to the most general Master Equation (not in Lindblad form ${ }^{1}$ ), I solve this last exactly (at a bath temperature $T=0$ ) obtaining an operatorial expression for $\rho_{S}(t)$. Moreover, in the contest of optical quantum system I derive an expression for $\rho_{S}(t)$ that is in accordance with the Carmichael's one, but, differently from that, the mine is applicable also when the Master equation is not in the Lindblad form in which cases Carmichael's solution is very often merely formal, as the same author underline in recent papers $[12,13]$. My mathematical tool, here called $N u D$ Theorem, is applicable to a wide class of systems, provided that they satisfy the hypothesis necessary to make it working. Optical physical systems well satisfy the conditions of $\mathrm{NuD}$ theorem's validity and in this contest I have studied, as example of monopartite system, a single mode cavity, and as example of multipartite systems, two two-level dipole-dipole interacting atoms and $N$ two-level not-directly-interacting atoms placed in fixed arbitrary point inside a loss cavity $[15,16]$. In the investigation of the monopar-

\footnotetext{
${ }^{1}$ Every Markovian Master Equation can be put in Lindblad form and this, in general, introduce simplification in the further calculations, but because of the difficulty to recast the equation in this form the results obtained are in general merely formal. For this reason I prefer to work with non-diagonal Markovian Master Equation, by virtue of which my solution is applicable to the more of the known open-system theoretical models -from matter-radiation interaction model to the spin-boson model-, more easily than other approaches.
} 
tite systems the novelty is constituted by the operatorial way to approach to the master equations of the systems, being already known in literature $[1,2,3,17]$ their dynamical properties. I reproduce for example the photocounting formula in order to appreciate the easyness of application of my method. Moreover I choose to analyze monopartite systems at a temperature $T=0$ to highlight that, differently to multipartite ones, spontaneous emission provokes decoherence phenomena that, inevitably, guides the first kind of systems to their ground states. On the contrary, multipartite systems can exhibit collective properties induced by the common reservoir $[15,16,18,19,20,21,22,23]$. This general feature already appeared in some fundamental papers $[24,18]$ in which the interaction between atomic dipoles, induced by electromagnetic field, could cause the decay of the multiatom system with two significantly different spontaneous emission rates, one enhanced and the other reduced.

In two recent papers, as an example of multipartite system, I have investigated the dynamics of a couple of spontaneously emitting two-level atoms, taking into account from the very beginning their dipole-dipole interaction and and $N$ twolevel not-directly-interacting atoms placed in fixed arbitrary point inside a loss cavity $[15,16]$. The result, not trivially expected, is that in such a condition the matter subsystem, because of the cooperation induced by energy loss mechanism, may be conditionally guided toward a stationary robust entangled state. The renewed interest toward entanglement concept reflects the consolidated belief that unfactorizable states of multipartite system provide an unreplaceable applicative resource, for example, in the quantum computing research area [25]. However, the realization of quantum computation protocols suffers of the difficulty of isolating a quantum mechanical system from its environment. In this sense the cited work are also aimed at proposing theoretical scheme to bypass decoherence manifestations, so taking its place among intense theoretical and experimental research of the last few years $[15,16,26,27,28,29,30,31,32]^{2}$.

The paper is structured as follow: in section II I report the principal step and approximation leading to the microscopic derivation of the Markovian Master Equation, putting in evidence some peculiar properties of it useful in order to demonstrate the $N u D$ Theorem $N$. In section III I solve the markovian master equation when $T=0$. In section IV I review the applications to old exemplary problem and to new, previous unresolved, problem. In section V I try to justify the obtained dynamical behaviour in terms of continuous measurement theory.

\footnotetext{
${ }^{2}$ Citations $[15,16]$ represent my first results about noise-induced entanglement. Even if they are obtained solving the coupled differential equation system of the block vector components describing the open system evolution, they can be considered the first $\mathrm{NuD}$ theorem application because the way in which I obtain the solution is always the same and it find its generalization in the $\mathrm{NuD}$ theorem.
} 


\section{Quantum Markovian Master Equation}

It is well know that under the Rotating Wave and the Born-Markov approximations the master equation describing the reduced dynamical behavior of a generic quantum system linearly coupled to an environment can be put in the form [1]

$$
\dot{\rho_{S}}(t)=-i\left[H_{S}+H_{L S}, \rho_{S}(t)\right]+D\left(\rho_{S}(t)\right),
$$

where $H_{S}$ is the hamiltonian describing the free evolution of the isolated system,

$$
\begin{gathered}
D\left(\rho_{S}(t)\right)=\sum_{\omega} \sum_{\alpha, \beta} \gamma_{\alpha, \beta}(\omega)\left(A_{\beta}(\omega) \rho_{S}(t) A_{\alpha}^{\dagger}(\omega)\right. \\
\left.-\frac{1}{2}\left\{A_{\alpha}^{\dagger}(\omega) A_{\beta}(\omega), \rho_{S}(t)\right\}\right), \\
H_{L S}=\sum_{\omega} \sum_{\alpha, \beta} S_{\alpha, \beta}(\omega) A_{\alpha}^{\dagger}(\omega) A_{\beta}(\omega) \\
S_{\alpha, \beta}(\omega)=\frac{1}{2 i}\left(\Gamma_{\alpha, \beta}(\omega)-\Gamma_{\beta, \alpha}^{*}(\omega)\right)
\end{gathered}
$$

and

$$
\gamma_{\alpha, \beta}(\omega)=\Gamma_{\alpha, \beta}(\omega)+\Gamma_{\beta, \alpha}^{*}(\omega),
$$

$\Gamma_{\alpha, \beta}(\omega)$ being the one-sided Fourier transforms of the reservoir correlation functions. Finally we recall that the operators $A_{\alpha}(\omega)$ and $A_{\alpha}^{\dagger}(\omega)$, we are going to define and whose properties we are going to explore, act only in the Hilbert space of the system.

Eq. (1) has been derived under the hypothesis that the interaction hamiltonian between the system and the reservoir, in the Schrödinger picture, is given by [1]

$$
H_{I}=\sum_{\alpha} A^{\alpha} \otimes B^{\alpha}
$$

that is the most general form of the interaction.

In the above expression $A^{\alpha}=\left(A^{\alpha}\right)^{\dagger}$ and $B^{\alpha}=\left(B^{\alpha}\right)^{\dagger}$ are operators acting respectively on the Hilbert space of the system and of the reservoir. The eq. (6) can be written in a slightly different form if one decomposes the interaction hamiltonian into eigenoperators of the system and reservoir free hamiltonian.

DEFINITION 1. Supposing the spectrums of $H_{S}$ and $H_{B}$ to be discrete (generalization to the continuous case is trivial) let us denote the eigenvalue of $H_{S}\left(H_{B}\right)$ by $\varepsilon(\eta)$ and the projection operator onto the eigenspace belonging to the eigenvalue $\varepsilon(\eta)$ by $\Pi(\varepsilon)(\Pi(\eta))$. Then we can define the operators:

$$
A_{\alpha}(\omega) \equiv \sum_{\varepsilon^{\prime}-\varepsilon=\omega} \Pi(\varepsilon) A_{\alpha} \Pi\left(\varepsilon^{\prime}\right)
$$




$$
B_{\alpha}(\omega) \equiv \sum_{\eta^{\prime}-\eta=\omega} \Pi(\eta) B_{\alpha} \Pi\left(\eta^{\prime}\right) .
$$

From the above definition we immediately deduce the following relations

$$
\begin{gathered}
{\left[H_{S}, A_{\alpha}(\omega)\right]=-\omega A_{\alpha}(\omega), \quad\left[H_{B}, B_{\alpha}(\omega)\right]=-\omega B_{\alpha}(\omega),} \\
{\left[H_{S}, A_{\alpha}^{\dagger}(\omega)\right]=+\omega A_{\alpha}^{\dagger}(\omega) \text { and }\left[H_{B}, B_{\alpha}^{\dagger}(\omega)\right]=+\omega B_{\alpha}^{\dagger}(\omega) .}
\end{gathered}
$$

An immediate consequence is that the operators $A_{\alpha}^{\dagger}(\omega>0)$ e $A_{\alpha}(\omega>0)$ raise and lower the energy of the system $S$ by the amount $\hbar \omega$ respectively and that the corresponding interaction picture operators take the form

$$
\begin{gathered}
e^{i H_{S} t} A_{\alpha}(\omega) e^{-i H_{S} t}=e^{-i \omega t} A_{\alpha}(\omega), \quad e^{i H_{B} t} B_{\alpha}(\omega) e^{-i H_{B} t}=e^{-i \omega t} B_{\alpha}(\omega), \\
e^{i H_{S} t} A_{\alpha}^{\dagger}(\omega) e^{-i H_{S} t}=e^{+i \omega t} A_{\alpha}^{\dagger}(\omega) \text { and } e^{i H_{B} t} B_{\alpha}^{\dagger}(\omega) B^{-i H_{B} t}=e^{+i \omega t} B_{\alpha}^{\dagger}(\omega) .
\end{gathered}
$$

Finally we note that

$$
A_{\alpha}^{\dagger}(\omega)=A_{\alpha}(-\omega) \text { and } B_{\alpha}^{\dagger}(\omega)=B_{\alpha}(-\omega) .
$$

Summing eq. (13) over all anergy differences and employing the completeness relation we get

$$
\sum_{\omega} A_{\alpha}^{\dagger}(\omega)=\sum_{\omega} A_{\alpha}(-\omega)=A_{\alpha} \quad \text { and } \quad \sum_{\omega} B_{\alpha}^{\dagger}(\omega)=\sum_{\omega} B_{\alpha}(-\omega)=B_{\alpha}
$$

The above positions enable us to cast the interaction hamiltonian into the following form

$$
H_{I}=\sum_{\alpha, \omega, \omega^{\prime}} A_{\alpha}(\omega) \otimes B_{\alpha}\left(\omega^{\prime}\right)=\sum_{\alpha, \omega, \omega^{\prime}} A_{\alpha}^{\dagger}(\omega) \otimes B_{\alpha}^{\dagger}\left(\omega^{\prime}\right)
$$

The reason for introducing the eigenoperator decomposition, by virtue of which the interaction hamiltonian in the interaction picture can now be written as

$$
H_{I}(t)=\sum_{\alpha, \omega, \omega^{\prime}} e^{-i\left(\omega+\omega^{\prime}\right) t} A_{\alpha}(\omega) \otimes B_{\alpha}\left(\omega^{\prime}\right),
$$

is that exploiting the rotating wave approximation, whose microscopic effect is to drop the terms for which $\omega \neq-\omega^{\prime}$, is equivalent to the schrodinger picture interaction hamiltonian:

$$
H_{I}=\sum_{\alpha, \omega} A_{\alpha}(\omega) \otimes B_{\alpha}(-\omega)=\sum_{\alpha, \omega} A_{\alpha}(\omega) \otimes B_{\alpha}^{\dagger}(\omega) .
$$


LEMMA 2. The Rotating Wave Approximation imply the conservation of the free energy of the global system, that is

$$
\left[H_{S}+H_{B}, H\right]=0
$$

\subsection{ProOF}

The necessary condition involved in the previous proposition is equivalent to the equation $\left[H_{S}+H_{B}, H_{I}\right]=0$ we are going to demonstrate.

$$
\begin{aligned}
{\left[H_{S}+H_{B}, H\right] } & =\left[H_{S}+H_{B}, H_{I}\right]=\left[H_{S}, H_{I}\right]+\left[H_{B}, H_{I}\right] \\
& =\sum_{\alpha, \omega}\left[H_{S}, A_{\alpha}(\omega)\right] \otimes B_{\alpha}^{\dagger}(\omega)+\sum_{\alpha, \omega} A_{\alpha}(\omega) \otimes\left[H_{B}, B_{\alpha}^{\dagger}(\omega)\right] \\
& =-\sum_{\alpha, \omega} \omega A_{\alpha}(\omega) \otimes B_{\alpha}(-\omega)+\sum_{\alpha, \omega} \omega A_{\alpha}(\omega) \otimes B_{\alpha}(-\omega)=0 .
\end{aligned}
$$

where we have made use of eq. $(9,10)$

LEMMA 3. The detailed balance condition in the thermodynamic limit imply [4]

$$
\gamma_{\alpha \beta}(\omega)=e^{-\beta \omega} \gamma_{\alpha \beta}(-\omega)
$$

where $\beta=\left(k_{B} T\right)^{-1}$

COROLLARY 4. Let us suppose the temperature of the thermal reservoir to be the absolute zero, on the ground of Lemma 2 immediately we see that

$$
\gamma_{\alpha \beta}(\omega<0)=0
$$

Let us now cast eq. (1) in a slightly different form splitting the sum over the frequency, appearing in eq. (2), in a sum over the positive frequencies and a sum over the negative ones so to obtain

$$
\begin{aligned}
& =\sum_{\omega>0, \alpha, \beta}^{D\left(\rho_{S}(t)\right)} \gamma^{\alpha, \beta}(\omega)\left(A^{\beta}(\omega) \rho_{S} A^{\alpha \dagger}(\omega)\right. \\
& \left.-\quad \frac{1}{2}\left\{A^{\beta \dagger}(\omega) A^{\alpha}(\omega), \rho_{S}\right\}\right) \\
& +\sum_{\omega>0, \alpha, \beta} \gamma^{\alpha, \beta}(-\omega)\left(A^{\alpha \dagger}(\omega) \rho_{S} A^{\beta}(\omega)\right. \\
& \left.-\frac{1}{2}\left\{A^{\alpha}(\omega) A^{\beta \dagger}(\omega), \rho_{S}\right\}\right),
\end{aligned}
$$


where we again make use of eq. (13). In the above expression we can recognize the first term as responsible of spontaneous and stimulated emission processes, while the second one takes into account stimulated absorption, as imposed by the lowering and raising properties of $A^{\alpha}(\omega)$. Therefore if the reservoir is a thermal bath at $T=0$ the corollary 4 tell us that the correct dissipator of the Master Equation can be obtained by suppressing the stimulated absorption processes in eq. (22).

\section{NuD Theorem}

We are now able to solve the markovian master equation when the reservoir is in a thermal equilibrium state characterized by $T=0$. We will solve a Cauchy problem assuming the factorized initial condition to be an eigenoperator of the free energy $H_{S}+H_{B}$. This hypothesis doesn't condition the generality of the found solution being able to extend itself to an arbitrary initial condition because of the linearity of the markovian master equation ${ }^{1}$.

NUD THEOREM 5. If eq. (1) is the markovian master equation describing the dynamical evolution of a open quantum system $S$, coupled to an environment $B$, assumed to be in the detailed-balance thermal equilibrium state characterized by a temperature $T=0$, and if the global system is initially prepared in a state $\rho(0)=$ $\rho_{B}(0) \rho_{S}(0)$ so that $\left(H_{S}+H_{B}\right) \rho(0)\left(H_{S}+H_{B}\right)=E_{L}^{2} \rho(0)$, where $E_{L}=E_{S}+E_{B}$ is the free energy of the global system then $\rho_{S}(t)$ is in the form of a Piecewise Deterministic Process [1], that is a process obtained combining a deterministic time-evolution with a jump process.

\subsection{ProOF}

The weak-coupling assumption is equivalent to $\rho(t)=\rho_{B}(0) \rho_{S}(t)$. The above equation can be used to derive the reduced density matrix $\rho(t)$ tracing over the environment degree of freedom. Let us choose a factorized base $B$ in the tensor product Hilbert space made of eigenvectors of $H_{S}$ and $H_{B}$

$$
B=\left\{\left|E_{B}, \lambda_{E_{B}}>\right| E_{S}, \lambda_{E_{S}}>\right\}
$$

where $\left\{E_{B}\right\}$ and $\left\{E_{S}\right\}$ define respectively the spectra of $H_{B}$ and $H_{S}$ and $\left\{\lambda_{E_{B}}\right\}$ and $\left\{\lambda_{E_{S}}\right\}$ their relative degenerations. Let us remember that we have made the semplificative hypotheses of discreteness of $\left\{E_{B}\right\}$ and $\left\{E_{S}\right\}$. In addition we

\footnotetext{
${ }^{1}$ It is out of relevance to consider initial condition having non-zero coherence between the environment and the system because it is not possible to resolve them in the reduced dynamics obtained tracing on the environment degrees of freedom.
} 
assume, also for easyness, that $\left\{E_{S}\right\}$ is bounded from below and made of isolated points. On the ground of these chioses the total density matrix can be written as

$$
\begin{array}{r}
\rho(t)=\sum_{E_{B}, \lambda_{E_{B}}, E_{S}, \lambda_{E_{S}}, E_{B}^{\prime}, \lambda_{E_{B}}^{\prime}, E_{S}^{\prime}, \lambda_{E_{S}}^{\prime}} \rho\left(E_{B}, \lambda_{E_{B}}, E_{S}, \lambda_{E_{S}}, E_{B}^{\prime}, \lambda_{E_{B}}^{\prime}, E_{S}^{\prime}, \lambda_{E_{S}}^{\prime}, t\right) \\
\left|E_{B}, \lambda_{E_{B}}>\right| E_{S}, \lambda_{E_{S}}><E_{B}^{\prime}, \lambda_{E_{B}}^{\prime}\left|<E_{S}^{\prime}, \lambda_{E_{S}}^{\prime}\right| .
\end{array}
$$

The Lemma 2. imposes a strong selection rule on the indices of the summation, that is :

$$
E_{S}+E_{B}=E_{S}^{\prime}+E_{B}^{\prime}
$$

by virtue of which the trace over the degrees of freedom of the environment, that can be written as

$$
\rho_{S}(t)=\sum_{E_{S}, \lambda_{E_{S}}, E_{S}^{\prime}, \lambda_{E_{S}}^{\prime}} \rho\left(E_{S}, \lambda_{E_{S}}, E_{S}^{\prime}, \lambda_{E_{S}}^{\prime}, t\right)\left|E_{S}, \lambda_{E_{S}}><E_{S}^{\prime}, \lambda_{E_{S}}^{\prime}\right|,
$$

is immediately obtained:

$$
\rho_{S}(t)=\sum_{E_{S}}\left(\sum_{\lambda_{E_{S}}, \lambda_{E_{S}}^{\prime}} \rho\left(E_{S}, \lambda_{E_{S}}, \lambda_{E_{S}}^{\prime}, t\right)\left|E_{S}, \lambda_{E_{S}}><E_{S}, \lambda_{E_{S}}^{\prime}\right|\right)=\sum_{E_{S}} \rho_{E_{S}}(t) .
$$

The ensemble $\left\{E_{S}\right\}$ can be put in biunivocal correspondence with the natural ensemble so that

$$
\rho_{S}(t)=\sum_{i=0}^{N} \rho_{i}(t)
$$

where $N$ is the natural index corresponding to the maximum of $\left\{E_{S}\right\}$. The operators $\rho_{i}(t)$ satisfy a big orthogonality condition that is

$$
\rho_{i}(t) \cdot \rho_{j}(t)=\rho_{i}^{2} \delta_{i j}
$$

The last two equations demonstrate that the evolution is a piecewise process (PP) or, equivalently, a statistical mixture of alternative generalized trajectories. These last are generalized respect to F.Petruccione and H.J.Carmochael approach, which leads to $\rho_{S}(t)=\sum_{i}\left|\psi_{i}><\psi_{i}\right|$. The last expansion, in terms of proper trajectories, is obtainable from the mine if and only if we are able to dygonalize the spectral correlation tensor, that is known to be always possible, but nobody is able to do it, with exception of few highly symmetrical systems. In order to demonstrate the formal equivalence between the two approaches we have to demonstrate that the alternative processes are deterministic (PDP) or, equivalently, that every of them is representable in the form of an evolutionary equation. 
On the ground of Lemma 2.1. and its corollary the markovian master equation at $T=0$ can be written as

$$
\begin{aligned}
\dot{\rho}_{S}(t)= & -i\left[H_{S}+H_{L S}, \rho_{S}(t)\right]+D\left(\rho_{S}(t)\right), \\
D\left(\rho_{S}(t)\right)= & \sum_{\omega>0} \sum_{\alpha, \beta} \gamma_{\alpha, \beta}(\omega)\left(A_{\beta}(\omega) \rho_{S}(t) A_{\alpha}^{\dagger}(\omega)\right. \\
& \left.-\frac{1}{2}\left\{A_{\alpha}^{\dagger}(\omega) A_{\beta}(\omega), \rho_{S}(t)\right\}\right) .
\end{aligned}
$$

Let us now substitute eq. (28) into eq. (30), so obtaining

$$
\begin{aligned}
& \sum_{i=0}^{N} \dot{\rho}_{i}(t)=-\frac{i}{\hbar} \sum_{i=0}^{N}\left[H_{0}, \rho_{i}(t)\right]+\sum_{i, \omega>0, \alpha, \beta} \gamma^{\alpha, \beta}(\omega)\left(A^{\beta}(\omega) \rho_{i} A^{\alpha \dagger}(\omega)\right. \\
& \left.-\frac{1}{2}\left\{A^{\beta \dagger}(\omega) A^{\alpha}(\omega), \rho_{i}\right\}\right) .
\end{aligned}
$$

$N$ being the natural index corresponding to the initial eigenvalue of $H_{S}{ }^{2}$. Let us observe that

$$
\sum_{\omega>0, \alpha, \beta} \gamma^{\alpha, \beta}(\omega) A^{\beta}(\omega) \rho_{0} A^{\alpha \dagger}(\omega)=0
$$

so that in the second summation the index $i$ starts from 1 and then the eq. (32) can be written as

$$
\begin{aligned}
\sum_{i=0}^{N-1} & {\left[\dot{\rho}_{i}(t)+\frac{i}{\hbar}\left[H_{0}, \rho_{i}(t)\right]\right.} \\
+ & \left.\sum_{\omega>0, \alpha, \beta} \gamma^{\alpha, \beta}(\omega)\left(A^{\beta}(\omega) \rho_{i+1} A^{\alpha \dagger}(\omega)+\frac{1}{2}\left\{A^{\beta \dagger}(\omega) A^{\alpha}(\omega), \rho_{i}\right\}\right)\right] \\
+ & {\left[\dot{\rho_{N}}(t)+\frac{i}{\hbar}\left[H_{0}, \rho_{N}(t)\right]+\sum_{\omega>0, \alpha, \beta} \gamma^{\alpha, \beta} \frac{1}{2}\left\{A^{\beta \dagger}(\omega) A^{\alpha}(\omega), \rho_{i}\right\}\right]=0 }
\end{aligned}
$$

Since the big orthogonality of $\left\{\rho_{i}\right\}$ the addenda in the above equation act on disjointed subspaces of the Hilbert space of $H_{S}$. This property implies that the above equation is verified if and only if the below system of differential coupled equations holds

\footnotetext{
${ }^{2} \mathrm{At} \mathrm{T}=0$ only spontaneous emission processes are involved in the dynamics, so that the initial eigenvalue of $H_{S}$ is the maximum permitted eigenvalue.
} 


$$
\begin{aligned}
& \dot{\rho}_{i}(t)=-\frac{i}{\hbar}\left[H_{0}, \rho_{i}(t)\right] \\
& +\sum_{\omega>0, \alpha, \beta} \gamma^{\alpha, \beta}(\omega)\left(A^{\beta}(\omega) \rho_{i+1} A^{\alpha \dagger}(\omega)-\frac{1}{2}\left\{A^{\beta \dagger}(\omega) A^{\alpha}(\omega), \rho_{i}\right\}\right) .
\end{aligned}
$$

It is immediate verify by substitution that

$$
\rho_{i}(t)=U(t) f_{i}(t) U^{\dagger}(t)
$$

where, in particular,

$$
f_{N}(t)=\rho_{N}(0)
$$

and $U(t)=e^{-\frac{i}{\hbar} B t}, U^{\dagger}(t)=e^{\frac{i}{\hbar} B^{\dagger} t}, B$ being

$$
B=H_{0}-\frac{i}{2 \hbar} \sum_{\omega>0, \alpha, \beta} \gamma^{\alpha \beta}(\omega) A^{\beta \dagger}(\omega) A^{\alpha}(\omega) \equiv H_{0}-\frac{i}{2 \hbar} H^{\prime},
$$

with $H^{\prime}$ hermitian. Finally,

$$
\begin{aligned}
f_{N-j} & (t)=\sum_{\omega^{\prime}, \alpha^{\prime}, \beta^{\prime}} \sum_{\omega^{\prime \prime}, \alpha^{\prime \prime}, \beta^{\prime \prime}} \ldots \sum_{\omega^{j}, \alpha^{j}, \beta^{j}} \gamma^{\alpha \beta}(\omega) \gamma^{\alpha^{\prime} \beta^{\prime}}\left(\omega^{\prime}\right) \gamma^{\alpha " \beta^{\prime \prime}}\left(\omega^{\prime \prime}\right) \ldots \gamma^{\alpha^{j} \beta^{j}}\left(\omega^{j}\right) \quad(39) \\
\times & \int_{0}^{t} \int_{0}^{t^{\prime}} \int_{0}^{t^{\prime \prime}} \ldots \int_{0}^{t^{j}} d t^{\prime} d t^{\prime \prime} \ldots d t^{j} U^{-1}\left(t^{\prime}\right) A^{\beta^{\prime}}\left(\omega^{\prime}\right) U\left(t^{\prime}\right) U^{-1}\left(t^{\prime \prime}\right) A^{\beta^{\prime \prime}}\left(\omega^{\prime \prime}\right) U\left(t^{\prime \prime}\right) \ldots \\
& U^{-1}\left(t^{j}\right) A^{\beta^{j}}\left(\omega^{j}\right) U\left(t^{j}\right) f_{N}\left(t^{j}\right) U^{\dagger}\left(t^{j}\right) A^{\alpha^{j} \dagger}\left(\omega^{j}\right) U^{\dagger-1}\left(t^{\prime \prime}\right) \ldots \\
& U^{\dagger}\left(t^{\prime \prime}\right) A^{\alpha^{\prime \prime} \dagger}\left(\omega^{\prime \prime}\right) U^{\dagger-1}\left(t^{\prime \prime}\right) U^{\dagger}\left(t^{\prime}\right) A^{\alpha^{\prime} \dagger}\left(\omega^{\prime}\right) U^{\dagger-1}\left(t^{\prime}\right), \quad j=1, \ldots, N \quad \text { - }
\end{aligned}
$$

This concludes the proof and, in addition, ensures that the dynamical processes, whose statistical mixture gives the open system stocastic evolution, are deterministic. This demonstrates that the evolution is representable as a Piecewise Deterministic Process (PDP) [1]. The found solution generalizes the PDPs introduced by H.J.Carmichael and formalized by F.Petruccione and H.P.Breuer. Actually, it is applicable also when the Markovian Master Equation isn't in the Lindblad form. This, as already highlighted, in general, introduces simplification in the further calculations, but because of the difficulty to recast the equation in this form the results obtained are in general merely formal. Tough the eq. (39) seems complicated to use it is a powerful predictive tool. I have tested it in a lot of contests. Two of my results are already published, others will be object of future papers. All of them contain the same (predictable?) result: multipartite systems, discarding the physical nature of the parts and of the environment, can exhibit entangled stationary states towards the system can be guided by a probabilistic scheme of measurement. 


\section{Applications}

The best test of a new mathematical tool is its ability to reproduce old results and to predict new results. In the contest of old exemplary problem I have reproduced:

- the photocounting formula $[33,8]$

- the enviroment-induced entanglement between two two-level not-direct-interacting atoms placed in fixed arbitrary points in the free space $[33,34,35,36]$

- Carmichael unravelling of the master equation $[33,5]^{1}$

Moreover I have tested the $\mathrm{NuD}$ theorem's predictive capability solving the dynamics of:

- two two-level dipole-dipole interacting atoms placed in fixed arbitrary points inside a single mode cavity in presence of atomic spontaneous emission and cavity losses [16].

- $\mathrm{N}$ two-level not-direct-interacting atoms placed in fixed arbitrary points inside a single mode cavity in presence of atomic spontaneous emission and cavity losses [15].

- A bipartite hybrid model, known as Jaynes-Cummings model, constituted by an atom and a single mode cavity linearly coupled and spontaneously emitting in the same environment (work in progress)

- Two harmonic oscillator linearly coupled and spontaneously emitting in the same environment (work in progress)

\section{Was the dynamical behaviour of markovian quantum systems predictable? The open system in an open-observed environment model}

The physical basis to answer this question is provided by continuous measurement theory by virtue of which we can regard the Markovian Master Equation as the evolutionary equation describing an open quantum system, whose environment is continuously monitored. Indirect quantum measurements scheme (Braginsky and Khalili, 1992, [14]) seems to go to the right direction because it theorizes the solution of dynamical problem, here analyzed, as statistical mixture of independent events referred to as non-selective measurement. An indirect quantum measurement can be viewed as consisting of three elements. The first element is the quantum system of interest and it is called quantum object. The second element is the so-called quantum probe on which the measurement is performed. The

\footnotetext{
${ }^{1}$ Carmichael interpretation of unravelling is made in a slight different way. He evidences the possibility of different unravelling about which I'm trying to better understand. Instead F.Petruccione and H.P.Breuer emphasize the non selective-level of measurement that imply an independent -from a particular choice of the environment measured observable-, unique unravelling. This way to think go to my same direction.
} 
third element of the scheme is a classical apparatus by which a measurement on the quantum probe is performed.

If we identify the quantum probe as the environment and replace the third element with a quantum measurement ideal device (characterized by its own Hilbert space) able to detect all the environment elementary excitations coming from the system and to make a click as evidence of the detection, it is possible to show that the Markovian Master Equation is also obtainable from the microscopic model describing this ideal full-quantum measurement scheme. The principal effect of this quantum measurement ideal device is to induce loosing of memory in the environment respect to the interaction with the system: every time the system give an excitation to the environment the device capture it. I name it MemoryCleaner (M-C).

Let us suppose that a $t=0$ the environment is in its ground state (thermal state at the temperature $T=0$ ), the $\mathrm{M}-\mathrm{C}$ is in its ground state ${ }^{1}$ and the system is in an arbitrary excited state and let us ask: how does they evolve? Two composite events may happen:

- the system does not give excitations to the environment; the M-C doesn't capture excitations;

- the system looses one or more than one excitation in the environment; the $\mathrm{M}-\mathrm{C}$ captures excitations.

In the first case the M-C will not make a click, the environment, the system and the $\mathrm{M}-\mathrm{C}$ resulting undisturbed by their mutual interactions. In the second case the $\mathrm{M}-\mathrm{C}$ will produce one or more than one click as evidence of the fact that the system has exchanged energy with the environment and the environment has interacted with the M-C. In this second case the state of the system has changed because of the interaction with the environment, the state of the $\mathrm{M}-\mathrm{C}$ has changed because of the interaction with the environment, instead the status of environment has been reset, respect to the coupling to the system, to its initial state because of the presence of $\mathrm{M}-\mathrm{C}$ device. The time involved in the reset process can be considered of the same order of the coherence time characterizing a markovian environment.

This qualitative analysis shows that the introduction of ideal $\mathrm{M}-\mathrm{C}$ realizes a thermal equilibrium condition maintaining the environment temperature constant and equal to zero (deviations from equilibrium can happen in a time scale so short respect to the time in which the system change appreciably its state that we can assume to look at the system evolution in a coarse-grained time scale assuming the environment evolution to happen over time which are not resolved).

From a microscopic point of view this situation is represented by the hamilto-

\footnotetext{
${ }^{1}$ The characterization of the spectrum of the $\mathrm{M}-\mathrm{C}$ is not relevant in this contest. One may thinks of a photoemissive source coupled to an environment interacting with one or more than one ideal photon detector $[1,5]$.
} 
nian

$$
H=H_{M-C}+H_{S}+H_{B}+H_{I}+H_{B, M-C}
$$

containing the free energy of the quantistic M-C $\left(H_{M-C}\right)$, of the system $\left(H_{S}\right)$ and of the environment $\left(H_{B}\right)$ and the interaction hamiltonians $\left(H_{I}\right.$ and $\left.H_{B, M-C}\right)$ describing respectively the coupling between the $\mathrm{M}-\mathrm{C}$ and the environment and the coupling between the system and the environment. The reduced density matrix of the system is obtainable by tracing over the environment and M-C degrees of freedom the corresponding Liouville equation

$$
\dot{\rho}(t)=-\frac{i}{\hbar}[H, \rho(t)]
$$

where $\rho(t)$ is the total density matrix belonging to the Hilbert space given by the tensor product of the Hilbert space of the system, of the environment and of the $\mathrm{M}-\mathrm{C}$.

The thermal equilibrium condition in the environment, realized by the M-C, ensure that

$$
\rho(t)=\rho_{B}(0) \rho_{S, M-C}(t)
$$

where $\rho_{B}(0)$ is the initial Gibbs state $(T=0)$ of the environment and $\rho_{S, M-C}$ is the density matrix describing the quantum evolution in the tensor product Hilbert space of the system and the M-C. The trace over the M-C degrees of freedom $\left(\operatorname{Tr}_{M C}\{\cdot\}\right)$ give

$$
\begin{array}{r}
\rho_{B}(0) \dot{\rho_{S}}(t)=-\frac{i}{\hbar}\left[H_{S}, \rho_{B}(0) \rho_{S}(t)\right]-\frac{i}{\hbar}\left[H_{B}, \rho_{B}(0) \rho_{S}(t)\right] \\
-\frac{i}{\hbar}\left[H_{I}, \rho_{B}(0) \rho_{S}(t)\right]-\frac{i}{\hbar} \operatorname{Tr}_{M C}\left\{\left[H_{B, M-C}, \rho(t)\right]\right\}
\end{array}
$$

and then the trace over environment degrees of freedom $\left(\operatorname{Tr}_{B}\{\cdot\}\right)$ give

$$
\begin{array}{r}
\dot{\rho_{S}}(t)=-\frac{i}{\hbar}\left[H_{S}, \rho_{S}(t)\right]-\frac{i}{\hbar} \operatorname{Tr}_{B}\left\{\left[H_{I}, \rho_{B}(0) \rho_{S}(t)\right]\right\} \\
-\frac{i}{\hbar} \operatorname{Tr}_{B}\left\{\operatorname{Tr}_{M C}\left\{\left[H_{B, M-C}, \rho(t)\right]\right\}\right\} .
\end{array}
$$

The last term in the above equation is zero. This equation has to describes a quantum system $\mathrm{S}$ coupled to a memory-cleaned environment $\mathrm{B}$. The environment characterization ensures that $\rho_{S}(t)$ satisfies the eq. (30) known as Markovian Master Equation. Actually, this equation takes into account both, the quantum M-C device and the environment through the Markov and the Born approximations 
that can be seen as the memory-cleaned environment approximation and the environment equilibrium state assumption. Moreover, the introduction of the ideal $\mathrm{M}-\mathrm{C}$, beside to give a full justification of Born-Markov approximation, induces to think that an almost memory-cleaned environment is modeled by a not-ideal M-C which limit case, a full memory environment, is modeled by a not-working M-C, a device unable to capture excitations (to interact with environment) or, equivalently, by a closed microscopic model containing only the system, the environment and their interaction. In this way exact open system dynamics can be obtained by eq. (40) just removing the $\mathrm{M}-\mathrm{C}$ and its interaction. On the ground of this considerations it is possible to divide the eq. (30) in three terms:

- we can recognize in the sandwich terms

$$
\sum_{\omega>0, \alpha, \beta} \gamma^{\alpha, \beta}(\omega) A^{\beta}(\omega) \rho_{i}(t) A^{\alpha \dagger}(\omega)
$$

the jump terms due to the $\mathrm{M}-\mathrm{C}$ device and describing the capture of an excitation of frequency $\omega$ and the consequent projection (jump) of the vector state into the subspace characterized by a free energy lower of $\omega$ than the previous of the measurement act.

- we can recognize in the free energy constant terms

$$
\left.-\frac{1}{2} \sum_{\omega>0, \alpha, \beta} \gamma^{\alpha, \beta}\left\{A^{\beta \dagger}(\omega) A^{\alpha}(\omega), \rho_{i}(t)\right\}\right)
$$

the effective dissipative part responsible of going to zero of populations and coherences of the excited states of the open system of interest and due to the open observed environment we are considering. In fact these terms introduce an imaginary frequency in the free-energy spectrum of $H_{S}$, so generating an exponential decaying of excited free eigenstates.

- we can recognize in the free energy Lamb-Shift terms

$$
\sum_{\omega, \alpha, \beta} S^{\alpha, \beta}\left[A^{\beta \dagger}(\omega) A^{\alpha}(\omega), \rho_{i}(t)\right]
$$

the environment-induced multipartite cooperation not-vanished by the loss of memory of the environment.

The found solution ( $\mathrm{NuD}$ theorem) tell us that the state of the system is a statistical mixture of the free energy system eigenoperators. This fact depends and it is consistent with the existence of the M-C measurement device because the act of measurement introduces a stochastic variable respect to which we can only predict the probability to have one or another of the possible alternative measures. These probabilities can be regarded as the weight of the possible alternative generalized trajectories and, analytically, they are given by the partial traces of the $\rho_{i}$. With 
this approach the dynamics has to be depicted as a statistical mixture of this alternative generalized trajectories.

Moreover the found trajectories evolve in time in a deterministic way: for example the trajectory relative to the initially excited system state is a shifted free evolution characterized by complex frequencies that means an exponential decaying free evolution. This statement may give the sensation that every system has to decay in its ground state because of the observed dynamics. It is in general not true. Actually, if the system is multipartite, it is possible that it admits excited and entangled equilibrium Decoherence Free Subspace (DFS) (so as it happens in a lot of known models), constituted by states on which the action of $H_{I}$ is identically zero and then, if the system, during evolution, passes through one of these states, the successive dynamics will be decoupled from the environment evolution. An equilibrium condition is reached in which entanglement is embedded in the system $[15,16]$.

What could ensure, for example, that an entangled decoherence-free state, if existing, has been generated? (For example) the number of click we hear in a period of time long enough respect spontaneous emission rate. Actually, if the numbers of the clicks is less than the numbers of initial excitations then we can say that it has been generated a decoherence-free state $[15,16,34]$.

If, on the contrary, the system is monopartite it is possible to demonstrate that the only possible DFS is generated by the ground state of the system so that a monopartite system will loose its internal coherence and the population of excited states because of the measurement and the consequent interaction with a memorycleaned environment, unable to induce cooperation among the parts: there exists only one part.

In this case the numbers of clicks has to be the same of initial system excitations. The M-C sounds as a quantum logic counter.

\section{Bibliography}

1. F.Petruccione, H.P.Breuer, The Theory of open quantum sistem, Oxford University Press (2002)

2. C.W.Gardiner, Quantum Noise, Springer-Verlag Berlin (1991)

3. W.H.Louiselle, Quantum statistical properties of radiation, Ed. John Wiley e Sons (1973)

4. R.Alicki, K.Lendi, Quantum Dynamical Semigroups and Applications, Lectures Notes in Physics V286, (Springer-Verlag 1987)

5. H.Carmichael, An Open System Approach to Quantum Optics, Lectures Notes in Physics m18, (Springer-Verlag 1993)

6. Y.Castin, K.Molmer, Phys. Rev. Lett. 74, 3772 (1995)

7. J.Dalibard, Y.Castin, K.Molmer, Phys. Rev. Lett. 68, 580 (1992)

8. E.B.Davies, Commun. Math. Phys. 15, 277 (1969)

9. M.D.Srinivas, E.B.Davies, Opt. Acta 28, 981 (1981) 
10. M.B.Plenio, P.L.Knight, Rev. Mod. Phys. 70, 101 (1998)

11. F.Petruccione, H.P.Breuer, Concepts and methods in the theory of open quantum systems systems, Lectures Notes in Physics V622, Springer-Verlag Berlin (2003)

12. H.J. Carmichael and K. Kim, Optics Commun. 179, 417 (2000)

13. J.P. Clemens, L. Horvath, B.C. Sanders, and H.J. Carmichael, Phys. Rev. A 68, 023809 (2003)

14. V.B.Braginsky, F.Ya.Khalili, Quantum Measurement, Cambridge University Press (1992)

15. S. Nicolosi, A. Napoli, A. Messina, quant-ph0306104 (2003), submitted to EPJD

16. S. Nicolosi, A. Napoli, A. Messina , F.Petruccione quant-ph0402211 (2004), axcepted on PRA

17. S.M.Barnett, P.M.Radmore, Methods in Theoretical Quantum Optics, Oxford Series in Optical and Imaging Science, 15 (2002)

18. C.Leonardi, F.Persico, G.Vetri, La rivista del Nuovo Cimento9(1986) 3,4

19. G.Benivegna A.messina, J.Mod.Opt. 36 (1989) 1205

20. M.Kozierowski, S.M.Chumakov, A.A.Mamedov, Journal of Mod. Opt. 40 (1993)453

21. B.W.Shore, P.L.Knight, Journal of Mod. Opt. 40 (1993)7

22. I.K.Kudryavstev, A.Lambrecht, H.Moya-Cessa, P.L.Knight, Journal of Mod. Opt. 40 (1993)8

23. S.J.D.Phoenix, S.M.Barnett, Journal of Mod. Opt. 40 (1993)6

24. Dicke, phys. rep. 93 (1954)99

25. M. A. Nielsen and I. L. Chuang, Quantum Computation and Quantum Information, Cambridge University Press, Cambridge, (2000).

26. Guo Ping Guo et al, Phys. Rev. A 65, 042102 (2002)

27. Lu-Ming Duan et al, Phys. Rev. A 58, (1998)

28. J.I.Cirac, P.Zoller, Phys. Rev. A 50, R2799 (1993)

29. Shneider, Milburn, Phys. Rev. A 65, 042107 (2002)

30. G.J.Yang, O.Zobay, P.Meystre, Phys. Rev. A 59, 4012 (1998)

31. E.Hagley, X.Maitre, G.Nogues, C.Wunderlich, M.Brune, J.M.Raimond, S.Haroche, Phys. Rev. Lett. 79, 1 (1997)

32. P.Foldi, M.G.Benedict, A.Czirjak, Phys. Rev. A 65, 021802 (2002)

33. S.Nicolosi, Soluzione operatoriale esatta di master equation ottiche generalizzate: applicazioni a problemi di interazione radiazione materia, Degree Thesis (2003), unpublished

34. Plenio, Huelga, Beige, Knight, Phys. Rev. A 59 (1999) 2468

35. T. Pellizzari, S.A. Gardiner, J.I. Cirac, P.Zoller, Phys. Rev. Lett. 75, 3788 (1995)

36. Z.Ficek, R.Tanas, quant-ph 0302124 